\title{
Interferon- $\beta$ and temozolomide combination therapy for temozolomide monotherapy-refractory malignant gliomas
}

\author{
HIROSHI KAWAJI, TSUTOMU TOKUYAMA, TOMOHIRO YAMASAKI, \\ SHINJI AMANO, NAOTO SAKAI and HIROKI NAMBA
}

\author{
Department of Neurosurgery, Hamamatsu University School of Medicine, Hamamatsu, Shizuoka 431-3192, Japan
}

Received February 13, 2015; Accepted March 24, 2015

DOI: $10.3892 / \mathrm{mco} .2015 .542$

\begin{abstract}
Interferon- $\beta$ (IFN- $\beta$ ) has been found to downregulate $\mathrm{O}^{6}$-methyl-guanine-DNA methyltransferase and sensitize glioma cells to chemoradiation therapy. The effectiveness of IFN- $\beta$ and temozolomide (TMZ) combination therapy for newly diagnosed glioblastomas was previously reported. However, there is no clinical report of recurrent of malignant gliomas treated with the combination of IFN- $\beta$ and TMZ. In the present study, we reported 7 cases of gliomas classified as uncontrollable with adjuvant TMZ monotherapy, who were then treated with IFN- $\beta$ and TMZ combination therapy. The magnetic resonance imaging findings and clinical symptoms improved in the majority of the cases, with tolerable adverse events and minimal residual disability. The overall survival (OS) time from the date of the initial surgery exceeded 13 months, suggesting that this combination therapy was successful in improving the prognosis of malignant gliomas refractory to adjuvant TMZ monotherapy.
\end{abstract}

\section{Introduction}

Malignant gliomas are the most common primary malignant brain tumors (1). Despite first-line treatment with surgical resection and concomitant radiotherapy (RT) and temozolomide (TMZ) therapy, malignant gliomas have a dismal prognosis. In particular, the median overall survival (OS) of glioblastoma multiforme (GBM), the most malignant phenotype, is only 14.6 months (2). The majority of GBM patients experience disease progression after a median progression-free survival (PFS) of 7-10 months; however, none of the salvage therapies for progressive cases have been found to clearly prolong survival (1).

Correspondence to: Professor Hiroki Namba, Department of Neurosurgery, Hamamatsu University School of Medicine, 1-20-1 Handayama, Higashi-ku, Hamamatsu, Shizuoka 431-3192, Japan

E-mail: hnamba@hama-med.ac.jp

Key words: temozolomide, recurrence, interferon- $\beta$, malignant glioma
The epigenetic silencing of the $\mathrm{O}^{6}$-methyl-guanine-DNA methyltransferase (MGMT) gene by promoter methylation was shown to prolong survival in patients with malignant gliomas (3). Interferon- $\beta$ (IFN- $\beta$ ) enhances malignant glioma chemosensitivity to TMZ by downregulating MGMT transcription via p53 induction $(4,5)$. On the basis of these reports, a multicenter phase 1 trial of combination therapy with IFN- $\beta$ and TMZ for newly diagnosed malignant gliomas (INTEGRA study) was conducted in Japan. This study demonstrated a prolonged median OS of 17.1 months with a 1-year PFS rate of $50 \%$, with good tolerability (6). This combination therapy has been reported to be more effective for cases with unmethylated MGMT promoter (7). There is an ongoing phase 2 study of this combination therapy; however, there are currently no clinical reports available on IFN- $\beta$ and TMZ combination therapy for recurrent malignant glioma cases, except one case report (8). The aim of the present study was to investigate the effectiveness and hematological toxicity of the IFN- $\beta$ and TMZ combination therapy for 7 recurrent glioma cases.

\section{Patients and methods}

Patients and prior treatment. A total of 30 newly diagnosed malignant glioma cases (WHO grade 3 or 4) were treated with conventional RT/TMZ therapy between 2007 and 2013 following surgical resection at the Department of Neurosurgery, Hamamatsu University School of Medicine (Hamamatsu, Shizuoka, Japan). Following completion of this therapy, 15 cases proceeded to receive adjuvant TMZ therapy. Although the tumors remained under control with the TMZ monotherapy in 2 cases at the time of the present study, recurrence was diagnosed with magnetic resonance imaging (MRI) in 13 cases. The adjuvant TMZ therapy was discontinued in 5 of the cases due to hematological or neurological complications and was changed to other regimens in 8 cases (IFN- $\beta+$ TMZ in 7 cases and a non-TMZ regimen in 1 case).

Study protocol. The study was approved by our institutional review board and all participants gave their written informed consent prior to the study onset.

The patients were administered IFN- $\beta$ ( $3 \times 10^{6} \mathrm{IU} /$ body $)$ on the first day and 150 or $200 \mathrm{mg} / \mathrm{m}^{2} \mathrm{TMZ}$ on days $1-5$ in cycles of 28 days. This protocol was the same with the adjuvant therapy of the INTEGRA study. We retrospectively investigated 
Table I. Characteristics of the patients who received IFN- $\beta$ and TMZ combination therapy.

\begin{tabular}{|c|c|c|c|c|c|c|c|c|c|}
\hline $\begin{array}{l}\text { Case } \\
\text { no. }\end{array}$ & $\begin{array}{l}\text { Age, } \\
\text { years }\end{array}$ & Gender & Pathology & $\begin{array}{l}\text { TMZ } \\
\text { cycle, } \\
\text { days }\end{array}$ & Re-op & $\begin{array}{c}\text { Number of } \\
\text { IFN- } \beta+ \\
\text { TMZ cycles }\end{array}$ & $\begin{array}{l}\text { Hematological } \\
\text { toxicity } \geq \text { grade } 3\end{array}$ & $\begin{array}{c}\text { KPS } \\
(\%)\end{array}$ & $\begin{array}{c}\text { OS } \\
\text { (months) }\end{array}$ \\
\hline 1 & 43 & $\mathrm{~F}$ & $\mathrm{AA}$ & 15 & - & 17 & - & 70 & 41 \\
\hline 2 & 42 & M & $\mathrm{AA} \rightarrow \mathrm{GBM}$ & 4 & + & 16 & Neutropenia & 80 & 26 \\
\hline 3 & 58 & $\mathrm{~F}$ & GBM & 3 & + & 57 & - & 50 & 60 \\
\hline 4 & 44 & $\mathrm{~F}$ & GBM & 40 & - & 18 & - & 80 & 57 \\
\hline 5 & 66 & M & GBM & 8 & + & 24 & - & 40 & 42 \\
\hline 6 & 56 & $\mathrm{~F}$ & GBM & 8 & - & 9 & Lymphopenia & 80 & 21 \\
\hline 7 & 68 & $\mathrm{M}$ & GBM & 2 & - & 7 & - & 0 & 13 \\
\hline
\end{tabular}

AA, anaplastic astrocytoma; GBM, glioblastoma multiforme; TMZ, temozolomide; Re-op, reoperation; IFN- $\beta$, interferon- $\beta$; KPS, Karnofsky performance status; OS, overall survival.



Figure 1. Time course of case 1 on brain magnetic resonance imaging (MRI) (upper and middle rows, T1-weighted images with contrast enhancement; lower row, fluid-attenuated inversion recovery images). (a) Initial images. The left frontal weakly enhanced tumor was diagnosed as anaplastic astrocytoma (AA). (b) There was no residual tumor detected following total resection and radiotherapy (RT)/temozolomide (TMZ) therapy. (c) At 22 months from the initial resection, a contrast-enhanced area appeared at the resection site (arrowhead) and the corpus callosum (arrow). (d) TMZ monotherapy was restarted but the enhanced areas enlarged. The therapeutic regimen was changed to interferon- $\beta$ (IFN- $\beta$ )/TMZ at 26 months after the initial resection. (e) At 29 months, the patient experienced severe convulsions, with enlargement of the enhanced area and severe perifocal edema on MRI. Corticosteroid therapy was initiated. (f) At 41 months ( 15 cycles of IFN- $\beta /$ TMZ), the enhanced area of the corpus callosum had disappeared, although residual lesions were observed at the anterior horn of the left lateral ventricle and the resection margin (stable disease).

OS and $\geq$ grade 3 hematological toxicities according to the Common Terminology Criteria for Adverse Events, version 4.0 (http://ctep.cancer.gov/protocolDevelopment/ electronic_applications/ctc.htm), in the 7 cases in which the treatment regimen was changed from TMZ monotherapy to IFN- $\beta$ and TMZ combination therapy (IFN- $\beta /$ TMZ therapy).

\section{Results}

Patient characteristics. The patient characteristics are summarized in Table I. Of the 7 patients, 3 were male and the mean age was 53.8 years (range, $42-68$ years). The histopathological diagnosis of the initial surgical specimen was anaplastic astrocytoma (AA) in 2 cases and GBM in 5 cases. The immunohistochemical examination revealed an isocitrate dehydrogenase-1 mutation in case 1 and increased MGMT expression in case 7; on fluorescence in situ hybridization, $1 \mathrm{p} 19 \mathrm{q}$ co-deletion was negative in all the cases. No adverse events occurred during the conventional RT/TMZ therapy in any of the cases. The patients were diagnosed as uncontrolled with adjuvant TMZ monotherapy according to the radiological findings and neurological observations. Tumor progression was 


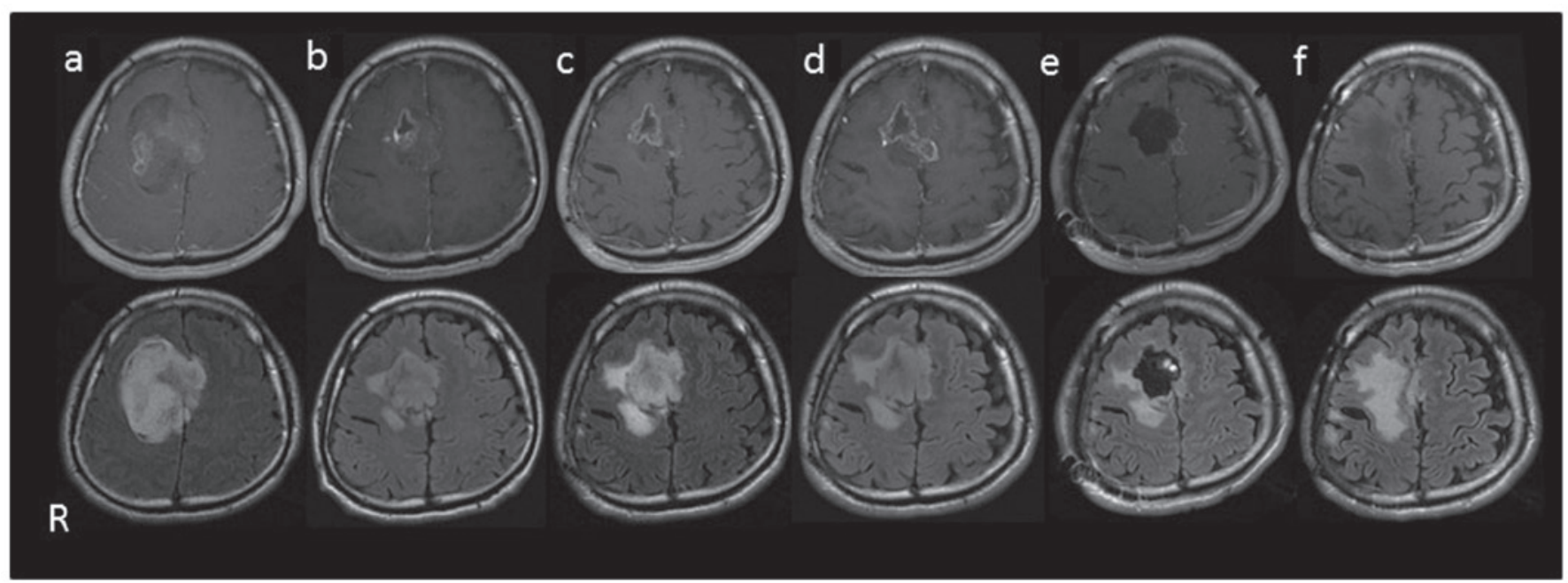

Figure 2. Time course of case 2 on brain magnetic resonance imaging (upper rows, T1-weighted images with contrast enhancement; lower row, fluid-attenuated inversion recovery images). (a) Initial images. The right frontal tumor was diagnosed as anaplastic astrocytoma (AA). (b) Following tumor resection and radiotherapy (RT)/temozolomide (TMZ) therapy, a small residual tumor was visible. (c) The TMZ monotherapy was discontinued for 5 months due to surgical site infection, but the tumor remained stable during this period. (d) The TMZ monotherapy was restarted, but the contrast-enhanced area enlarged. (e) The tumor was again removed and diagnosed as glioblastoma multiforme (GBM). The treatment regimen was changed to interferon- $\beta$ (IFN- $\beta$ )/TMZ. (f) The patient has been continuing this combination therapy without disease recurrence or significant disability.

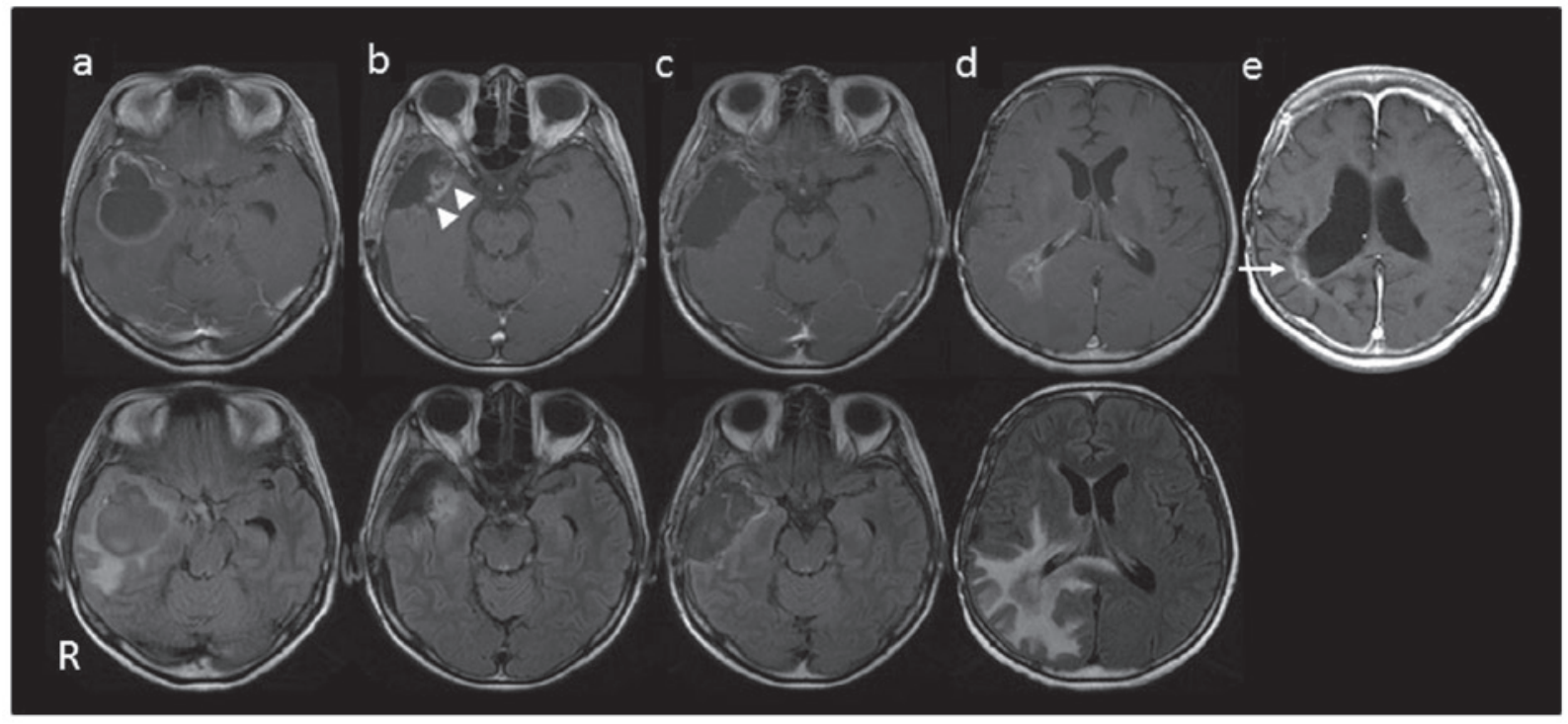

Figure 3. Time course of case 3 on brain magnetic resonance imaging (upper rows, T1-weighted images with contrast enhancement; lower row, fluid-attenuated inversion recovery images). (a) Initial images. The right temporal lobe cystic tumor was diagnosed as glioblastoma multiforme (GBM). (b) After 3 cycles of adjuvant temozolomide (TMZ) monotherapy, a contrast-enhanced area appeared at the resection site (arrow heads). (c) The recurrent tumor was completely resected and again diagnosed as GBM. (d) At 16 months after the initial resection [ 9 months after the initiation of interferon- $\beta$ (IFN- $\beta$ )/TMZ therapy], an enhanced area and perifocal edema appeared around the right lateral ventricle, in addition to the initial tumor region. (e) The enhanced area gradually decreased. At the time of the study (60 months after the initial resection and 53 months after initiation of IFN- $\beta / T M Z$ ), a small enhanced area was detectable, but this region remained stable over a long period of time (arrow), with no detectable tumor recurrence in the initial tumor region.

confirmed in 3 cases by the second operation (cases 2, 3 and 5) and the histopathological diagnosis was changed from AA to GBM in one case (case 2). The mean cycles of the adjuvant TMZ monotherapy period was 11.4 cycles (range, $2-40$ cycles).

The IFN- $\beta /$ TMZ therapy was performed for 7-57 cycles (mean, 21.1 cycles); $\geq$ grade 3 hematological toxicities occurred in 2 cases (cases 2 and 6) (Table I). Case 7 succumbed to the disease at 13 months from the initial surgery $(8$ months from the initiation of IFN- $\beta / \mathrm{TMZ}$ therapy); the other 6 cases remained alive at the time of the study, with an OS of
$>21$ months, although contrast-enhanced regions on MRI were still observed (stable disease). Three representative cases are described below.

Case 1 (Fig. 1). A 43-year-old woman with a left frontal AA (Fig. 1a) underwent complete resection of the tumor, with conventional RT/TMZ therapy and 12 cycles of adjuvant TMZ monotherapy (Fig. 1b). At 9 months, contrast-enhanced areas on MRI appeared at the resection site and the corpus callosum (Fig. 1c, arrowhead and arrow, respectively) with 
perifocal edema. TMZ monotherapy was restarted, but the enhancement area enlarged (Fig. 1d). The treatment regimen was then changed to IFN- $\beta / T M Z$. The patient experienced severe convulsions 3 months later, with enlargement of the enhanced area and severe perifocal edema on MRI (Fig. 1e). Corticosteroid therapy was initiated and the MRI findings (Fig. 1f) and clinical symptoms gradually improved. The patient is continuing the IFN- $\beta /$ TMZ therapy (has received a total of 17 cycles), with only mild right-sided motor weakness, and continues to work as a housekeeper [Karnofsky performance status (KPS), 70\%]. There was no pathological confirmation of the enhanced areas performed at the time of enlargement in this case.

Case 2 (Fig. 2). A 42-year old man with a right frontal AA (Fig. 2a) underwent maximal surgical removal of the tumor, with conventional RT/TMZ therapy and 2 cycles of adjuvant TMZ monotherapy (Fig. 2b). Due to surgical site infection, the TMZ therapy was discontinued for 5 months, during which time the tumor remained stable (Fig. 2c). The TMZ monotherapy was restarted, but the residual tumor enlarged after 2 cycles (Fig. 2d). The tumor was again removed (Fig. 2e) and diagnosed as GBM. The treatment regimen was then changed to IFN- $\beta /$ TMZ. At the time of this study, the patient had been continuing this combination therapy without disease recurrence or significant disability (KPS, $80 \%$; IFN- $\beta / \mathrm{TMZ}$ therapy, 16 cycles) (Fig. 2f).

Case 3 (Fig. 3). A 58-year-old woman with a right temporal GBM (Fig. 3a) underwent tumor resection. Following conventional RT/TMZ therapy and 3 cycles of adjuvant TMZ monotherapy, a contrast-enhanced area on MRI appeared at the resection site (Fig. 3b, arrowheads). The tumor was again removed (Fig. 3c) and IFN- $\beta /$ TMZ was initiated. The enhanced lesion with perifocal edema enlarged over several months (Fig. 3d) and then gradually decreased in size (Fig. 3e, arrow). The patient exhibited no neurological deficits at the time of the second surgery; however, the higher cortical function gradually decreased over several months (KPS, 50\%; IFN- $\beta /$ TMZ therapy, 57 cycles).

\section{Discussion}

In the present study, we reported the cases of 7 patients who had been refractory to adjuvant TMZ monotherapy and were treated with IFN- $\beta / \mathrm{TMZ}$. The rate of $\geq$ grade 3 hematological toxicity was $28.5 \%$ ( $2 / 7$ cases), which was higher compared with that observed in the INTEGRA study (6.7-13.3\%) (6); however, no patient discontinued the combination therapy due to hematological toxicity. The IFN- $\beta /$ TMZ therapy was, therefore, well tolerated. As shown in Table I, only 1 patient succumbed to the disease 13 months after the initial diagnosis, whereas the remaining 6 patients survived longer than 21 months. In addition, 4 patients maintained a KPS of $\geq 70 \%$ at the time of the study, with stable disease on MRI. It is considered that the addition of IFN- $\beta$ may suppress tumor growth and improve the prognosis of the patients with malignant gliomas refractory to TMZ monotherapy.

One of the limitations of this study is that tumor progression was confirmed by the second operation in only 3 cases. In case 7, IFN- $\beta$ was added at only 2 months after conventional radiation and TMZ therapy, due to the marginal increase of the contrast-enhanced region, which may be attributable to treatment-related changes. Additional imaging modalities, such as positron emission tomography with amino acid tracers, may be required for distinguishing tumor progression from treatment-related changes.

It is considered that IFN- $\beta$ potentiates the antitumor effect of TMZ by downregulating MGMT expression $(5,9)$. However, a recent study demonstrated that the expression of MGMT did not differ between the initial and the recurrent surgical specimens, unlike the expression of mismatch repair proteins, which decreases in the recurrent tumors (10). Therefore, increased expression of MGMT and also deficiency of the mismatch repair protein system and/or existence of glioma stem cells may be the mechanisms underlying glioma resistance to TMZ (11).

IFN- $\beta$ monotherapy induces apoptosis and exerts antiangiogenic effects in gliomas $(12,13)$. It has also been reported that IFN- $\beta$ suppresses the proliferation and self-renewal of glioma stem cells and, therefore, tumorigenesis (14), and that IFN- $\beta$ enhances the effect of chemoradiation therapy in glioma stem cells independently of MGMT expression (15). These pleiotropic antitumor effects of IFN- $\beta$ may have improved the prognosis of the TMZ-refractory cases presented in this study.

In conclusion, 7 malignant glioma cases, classified as uncontrollable with adjuvant TMZ monotherapy, were treated with IFN- $\beta / T M Z$. Suppression of further tumor growth and prolonged survival were achieved in the majority of the cases, suggesting that the IFN- $\beta / T M Z$ therapy was effective. Therefore, the adjuvant chemotherapy following conventional RT and TMZ therapy was changed from TMZ monotherapy to IFN- $\beta /$ TMZ combination therapy.

\section{References}

1. Omuro A and DeAngelis LM: Glioblastoma and other malignant gliomas: A clinical review. JAMA 310: 1842-1850, 2013.

2. Ohka F, Natsume A and Wakabayashi T: Current trends in targeted therapies for glioblastoma multiforme. Neurol Res Int 2012: 878425, 2012.

3. Hegi ME, Diserens AC, Gorlia T, et al: MGMT gene silencing and benefit from temozolomide in glioblastoma. N Engl J Med 352: 997-1003, 2005.

4. Natsume A, Ishii D, Wakabayashi T, Tsuno T, Hatano H, Mizuno $\mathrm{M}$ and Yoshida J: IFN-beta down-regulates the expression of DNA repair gene MGMT and sensitizes resistant glioma cells to temozolomide. Cancer Res 65: 7573-7579, 2005.

5. Natsume A, Wakabayashi T, Ishii D, Maruta H, Fujii M, Shimato S, Ito M and Yoshida J: A combination of IFN-beta and temozolomide in human glioma xenograft models: Implication of p53-mediated MGMT downregulation. Cancer Chemother Pharmacol 61: 653-659, 2008.

6. Wakabayashi T, Kayama T, Nishikawa R, et al: A multicenter phase I trial of combination therapy with interferon- $\beta$ and temozolomide for high-grade gliomas (INTEGRA study): The final report. J Neurooncol 104: 573-577, 2011.

7. Motomura K, Natsume A, Kishida Y, Higashi H, Kondo Y, Nakasu Y, Abe T, Namba H, Wakai K and Wakabayashi T: Benefits of interferon $\beta$ and temozolomide combination therapy for newly diagnosed primary glioblastoma with the unmethylated MGMT promoter: A multicenter study. Cancer 117: 1721-1730, 2011.

8. Fujimaki T, Ishii $\mathrm{H}$, Matsuno A, Arai $\mathrm{H}$ and Nakagomi T: Effectiveness of interferon-beta and temozolomide combination therapy against temozolomide-refractory recurrent anaplastic astrocytoma. World J Surg Oncol 5: 89, 2007. 
9. Park JA, Joe YA, Kim TG and Hong YK: Potentiation of antiglioma effect with combined temozolomide and interferon- $\beta$. Oncol Rep 16: 1253-1260, 2006.

10. Felsberg J, Thon N, Eigenbrod S, et al; German Glioma Network: Promoter methylation and expression of MGMT and the DNA mismatch repair genes MLH1, MSH2, MSH6 and PMS2 in paired primary and recurrent glioblastomas. Int J Cancer 129: 659-670, 2011.

11. Johannessen TC and Bjerkvig R: Molecular mechanisms of temozolomide resistance in glioblastoma multiforme. Expert Rev Anticancer Ther 12: 635-642, 2012.

12. Saito R, Mizuno M, Hatano M, Kumabe T, Yoshimoto $T$ and Yoshida J: Two different mechanisms of apoptosis resistance observed in interferon-beta induced apoptosis of human glioma cells. J Neurooncol 67: 273-280, 2004.
13. Hong YK, Chung DS, Joe YA, Yang YJ, Kim KM, Park YS, Yung WK and Kang JK: Efficient inhibition of in vivo human malignant glioma growth and angiogenesis by interferon-beta treatment at early stage of tumor development. Clin Cancer Res 6: 3354-3360, 2000.

14. Yuki K, Natsume A, Yokoyama H, Kondo Y, Ohno M, Kato T, Chansakul P, Ito M, Kim SU and Wakabayashi T: Induction of oligodendrogenesis in glioblastoma-initiating cells by IFN-mediated activation of STAT3 signaling. Cancer Lett 284: 71-79, 2009.

15. Happold C, Roth P, Silginer M, Florea AM, Lamszus K, Frei K, Deenen R, Reifenberger G and Weller M: Interferon- $\beta$ induces loss of spherogenicity and overcomes therapy resistance of glioblastoma stem cells. Mol Cancer Ther 13: 948-961, 2014. 